\title{
An injection needle as a cause of aspiration pneumonia in a male HIV(+) intravenous drug user
}

\author{
Krzysztof A. Simon ${ }^{1,2}$, Monika Pazgan-Simon ${ }^{1,2}$, Marcin Czarnecki ${ }^{2,3}$, Justyna Janocha-Litwin ${ }^{1,2}$ \\ ${ }^{1}$ Department of Infectious Diseases and Hepatology, Wroclaw Medical University, Wroclaw, Poland \\ ${ }^{2}$ I Department of Infectious Diseases, Regional Specialist Hospital, Wroclaw, Poland \\ ${ }^{3}$ Department of Infectious Diseases, Liver Diseases and Acquired Immune Deficiencies, Wroclaw Medical University, Wroclaw, Poland
}

\begin{abstract}
We report a case of a 56-year-old man with a long-term history of intravenous drug use who presented with 5-month history of cough and recurrent pneumonia. Except chronic HIV and hepatitis $\mathrm{C}$ virus $(\mathrm{HCV})$ infections there was no other significant medical history and no recollection of any foreign body aspiration. Repeated chest X-ray in many medical institutions showed only typical mild recurrent inflammatory changes in the right lower lobe. Using a flexible bronchoscopes we found and removed the injection needle. We observed rapid recovery and the patient was discharged from hospital 5 days later in good condition. Foreign body aspiration is rarely the cause of pneumonia and most often occurs in children. In patients infected with HIV due to immunodeficiency, with high risk of opportunistic infections and other AIDS-defining illnesses, iatrogenic etiology of lung pathology is rarely taken into account. Although the aspiration of a foreign body rarely causes recurrent pneumonia, in intravenous drug-dependent patients, it should be considered in the differential diagnosis, even if the results of repeated chest X-rays are negative. Radiography is the best method for the imaging of metallic foreign bodies. However, foreign bodies that retain X-ray radiation are detected in only approximately $25 \%$ of patients who have inhaled an object.
\end{abstract}

HIV AIDS Rev 2018; 17, 1: 61-63 DOI: https://doi.org/10.5114/hivar.2018.73342

Key words: HIV infection, iatrogenic pneumonia, foreign body aspiration.

\section{Introduction}

Foreign body aspiration is rarely the cause of pneumonia and most often occurs in children. In patients infected with HIV due to immunodeficiency, the risk of opportunistic infections and other AIDS-defining illnesses is very high, but iatrogenic etiology of lung pathology is rarely taken into account. Determining the proper diagnosis may be difficult if the patient's medical history shows no evidence suggesting the possibility of aspiration of a foreign body.

\section{Case report}

A 56-year-old man infected with HIV, addicted to intravenously taken opiates, was admitted to our Department of Infectious Diseases due to persistent dry cough, chest pain, and fever increasing for about 14 days.

The medical history revealed that the patient over the last thirty years had administered intravenously initially "compote" and then heroin in recent years. HIV co-infected with hepatitis $\mathrm{C}$ virus (HCV) was diagnosed in 2005. Despite active 
addiction, the man for six years took the antiretroviral drugs saquinavir/ritonavir, lamivudine and zidovudine. RNA not revealed from the beginning of HIV therapy indicated good compatibility with the therapy. The patient was addicted to smoking tobacco (for about 35 years, 20 cigarettes/day).

Intravenous injections of addictive drugs were in the past the cause of the patient's complications such as phlegmonous inflammation of the inguinal nodes, deep vein thrombosis of the left thigh and chronic venous insufficiency of the lower limbs. On 1 February 2015 the vessel puncture in the left axillary area for the purpose of injecting heroin was followed by massive bleeding from the groin. The bleeding was medicated in the emergency department where, by means of angio-CT, bleeding from the iliac and femoral artery, aneurysm and arteriovenous fistula were ruled out. As the patient at the same time reported complaints of weakness, shortness of breath, and cough with expectoration of purulent sputum, and X-ray examination showed inflammatory changes in the area of the lower lobe of the right lung, the patient was hospitalized in the Department of Internal Diseases and Hypertension in Nicolaus Copernicus Hospital in Gdansk. The patient was successfully treated with amoxicillin with clavulanic acid. Erythrocyte concentrate was transfused because of anemia and after the improvement of the general condition the patient was discharged with a recommendation to start the therapy in the addiction treatment center.

In January 2015, he came to the addiction treatment center MONAR in Milejowice near Wroclaw. On admission, the patient was in good general condition, except for mild cough, without significant discomfort. Physical examination revealed no deviation from the standard. In early March, the patient reported increased cough with expectoration of a small amount of clear sputum and periodically increased sweating at night, despite the absence of fever. Auscultation revealed quiet crackles at the base of the right lung. Chest X-ray showed a small amount of streaky densities in the right cardiophrenic angle possibly corresponding

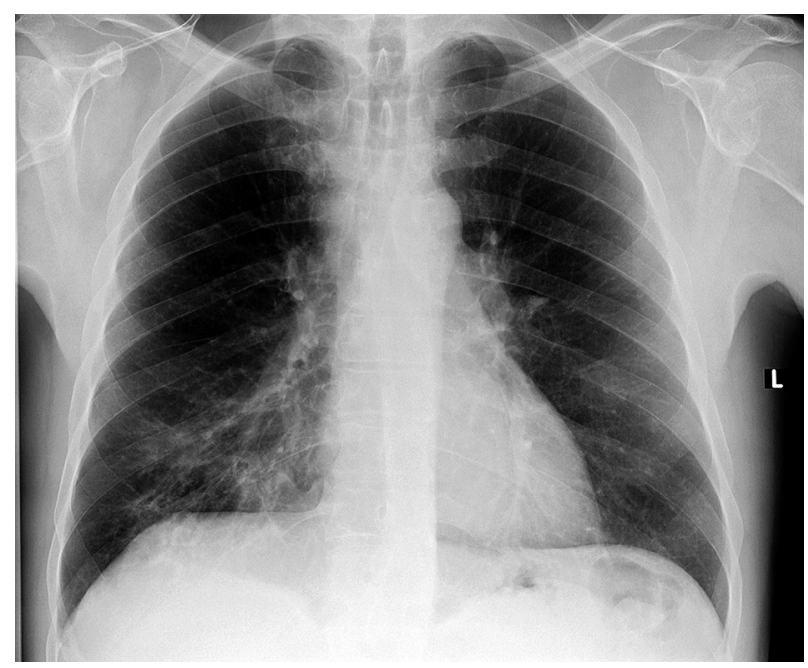

Figure 1. Chest X-ray, posteroanterior view to inflammatory changes. Due to the medical history with cigarette smoking, in the absence of fever and laboratory markers of inflammation (WBC, CRP normal), exacerbation of chronic obstructive pulmonary disease (COPD) was assumed as a possible cause of the ailment; spirometry was recommended (the patient did not make it) and the patient received inhaled steroids and bromhexine, obtaining relief of symptoms. After another two months, the patient again reported recurrence of tiring, productive cough, intensified in the morning and evening. There was no fever, and chest auscultation was unchanged. Spirometry was ordered once again along with serological tests for infection of Chlamydophila pneumoniae and Mycoplasma pneumoniae as well as C-reactive protein (CRP) and the number of CD4(+) cells. Three days later, the patient came to the doctor due to a sudden increase in body temperature to $39^{\circ} \mathrm{C}$ and a sore throat. The nature of the cough had not changed. The patient was diagnosed with viral pharyngitis. Paracetamol and topical chlorhexidine were recommended. However, due to persistent fever and further deterioration of well-being, after seven days, the patient was referred to the Department of Infectious Diseases WSS of Gromkowski in Wroclaw (July 2015).

On admission to the hospital the patient's general condition was good despite the fever up to $39^{\circ} \mathrm{C}$. Auscultation revealed discrete crackles at the base of the right lung. Laboratory tests drew attention to leukocytosis $\left(20.41 \times 10^{3} / \mu \mathrm{l}\right)$, the predominance of neutrophils $\left(19.38 \times 10^{3} / \mu \mathrm{l}\right)$, and concentration of CRP $(171 \mathrm{mg} / \mathrm{l})$. The X-ray of lungs revealed a peribronchial parenchymal density in the lower right lung field and prominent pulmonary hila of both lungs (Fig. 1). The sputum was subjected to bacterioscopic examination, cultures and molecular studies for presence of Mycobacterium tuberculosis. The differential diagnosis took into account streptococcal pneumonia, tuberculosis, atypical pneumonia and exacerbation of COPD. Tuberculosis is one of the most frequently diagnosed diseases in the group of $\operatorname{HIV}(+)$ among

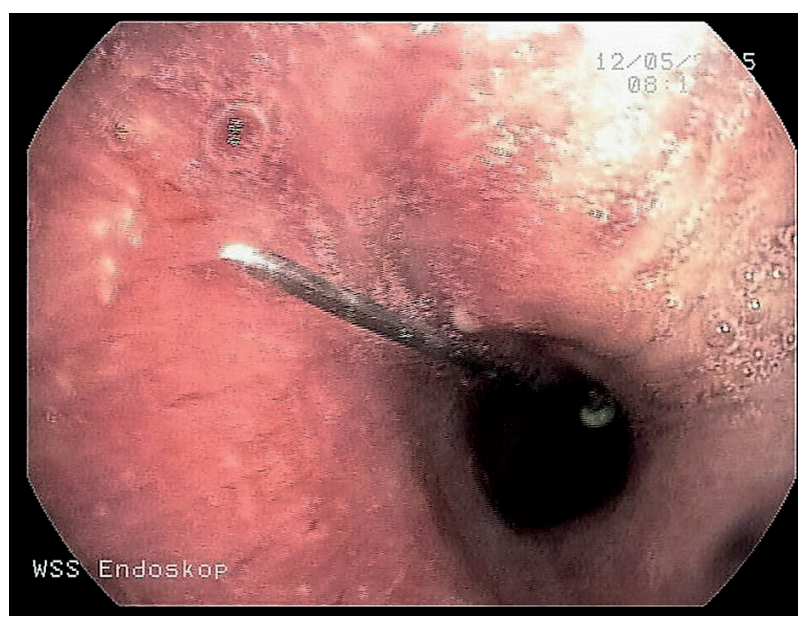

Figure 2. $40 \mathrm{~mm}$ long (21 gauge) injection needle inside the lobar bronchus in the lower lobe of the right lung 
addicted people hospitalized due to lung pathology [1]. Suspicion of pulmonary tuberculosis was supported by drug addiction, HIV infection, as well as the low number of lymphocytes CD4(+) - 228 cells/ $\mu$ (HIV - RNA PCR undetectable). Bronchoscopy revealed in the lobar bronchus in the lower lobe of the right lung, in addition to a small amount of muco-purulent secretions, unexpectedly a moving longitudinal artifact covered with brown deposits, which turned out to be an injection needle positioned with its blade upwards (Fig. 2). Otherwise, airways and mucous of other bronchi available for examination were normal. Using a flexible bronchofiberoscope (PENTAX EB 1975K) with $2.8 \mathrm{~mm}$ biopsy channel and thin biopsy forceps with windows (PENTAX KW1815), after repeated attempts, the sharp tip of the injection needle was locked in one of the windows of the forceps. Afterwards, the forceps were closed and the needle, under constant video control, was safely removed from the bronchial tree, without mucosal damage.

Results of tests for infection with Mycobacterium tuberculosis remained negative. Antibodies against Chlamydophila pneumoniae - IgG 41.3 AU/ml (positive > $18 \mathrm{AU} / \mathrm{ml}$ ), IgA $90.8 \mathrm{AU} / \mathrm{ml}$ (positive $>18 \mathrm{AU} / \mathrm{ml}$ ). Antibodies against $M y c o-$ plasma pneumoniae - IgM $0.2 \mathrm{AU} / \mathrm{ml}$ (negative $<0.9 \mathrm{AU} / \mathrm{ml}$ ) IgG 35.8 (positive $>18 \mathrm{AU} / \mathrm{ml}$ ). It was concluded that the pneumonia resulted from a foreign body in the bronchus in the form of the injection needle.

\section{Outcome and follow-up}

The patient was treated with amoxicillin and clarithromycin with clavulanic acid, which gave, after removal of the foreign body, fast relief of symptoms, improvement of the general condition of the patient and the regression of inflammatory changes in chest radiographs. The patient currently remains in good condition.

\section{Discussion}

The case study raises two important questions: 1) How and when did the injection needle get into the lobar bronchus of the lower lobe of the right lung? 2) Why was the metallic foreign body not seen in any of the radiographs?

An answer to the first question was provided by an insightful conversation with the patient. After injection of the drug, the patient always repeated the same series of steps, in his opinion, to reduce the risk of accidental transmission of HIV to other persons. Immediately after injection of the drug, the man removed the needle from the syringe and put it to his lips - the cap towards the interior of the mouth, the blade towards the outside, so as not to get hurt. At this time, he would destroy the syringe, so that it could not be subsequently re-used by anyone. After the destruction of the syringe, he took the needle out of his mouth and broke it. He threw the destroyed equipment into a sewage drain.

In December 2014, after the injection of the drug and with the needle inserted into his mouth, the man lost con- sciousness for a short time and after recovery he did not find the needle. Retrospectively, the patient did not rule out the possibility of aspiration of the needle. Such a course of events seems to be confirmed by the location of the foreign body in the lung - the lobar bronchus of the lower lobe of the right lung - as well as the position of the needle in the bronchus, with the blade upwards. According to the authors of a meta-analysis of aspiration pneumonia in adults, aspirated objects usually took such a location [2]. While staying at the addiction treatment center from mid-January 2015, the patient did not take drugs. Five months passed from the event to the diagnosis, and three routine X-ray chest radiographs were performed during that time without finding a metallic foreign body. Radiography is the best method for the imaging of metallic foreign bodies; however, foreign bodies that retain X-ray radiation are detected in only approximately $25 \%$ of patients who have inhaled an object $[3,4]$. Sometimes it is not even possible to identify a metallic object located in the tissues by conventional X-ray techniques [5]. Some authors emphasize the greater value of computed tomography in the detection of foreign objects in the lungs [2]. This was not done in this case. Bronchoscopy definitely has the highest sensitivity in the detection of foreign bodies in the bronchi. It was confirmed by our observations [3]. A similar case was described by Shah et al.; however, the foreign body revealed in the chest X-ray was removed only by a thoracic surgeon [6].

Although the aspiration of a foreign body rarely causes recurrent pneumonia, in intravenous drug-dependent patients, it should be considered in the differential diagnosis, even if the results of repeated chest X-rays are negative.

\section{Conflict of interest}

The author's declared no potential conflicts of interest with respect to the research, authorship, and/or publication of this article.

\section{References}

1. World Health Organization. 2013. Global Tuberculosis Report; http:// apps.who.int/iris/bitstream/10665/91355/1/9789241564656_eng. pdf.

2. Sehgal S, Dhooria S, Ram B, et al. Foreign Body Inhalation in the Adult Population: Experience of 25,998 Bronchoscopies and Systematic Review of the Literature. Respir Care 2015; 60: 1438-1448.

3. Ingraham CR, Mannelli L, Robinson JD, et al. Radiology of foreign bodies: how do we image them? Emerg Radiol 2015; 22: 425-430.

4. Orgill RD, Pasic TR, Peppier WW, et al. Radiographic evaluation of aspirated metallic foil foreign bodies. Ann Otol Rhinol Laryngol 2005; 114: 419-424.

5. Pinto A, Scaglione M, Pinto F, et al. Tracheobronchial 32 aspiration of foreign bodies: current indications for emergency plain chest radiography. Radiol Med (Torino) 2006; 111: 497-506.

6. Shah AR, Smyth L, Tolan M, et al. Haemoptysis in an intravenous drug user: injection needle impacted in the left main bronchus. BMJ Case Rep 2014; doi: 10.1136/bcr-2013-203336. 
